\title{
Racial and Weight Discrimination Associations with Pain Intensity and Pain Interference in An Ethnically Diverse Sample of Adults with Obesity: A Baseline Analysis of the Clustered Randomized-Controlled Clinical Trial the Goals for Eating and Moving (GEM) Study
}

Ericka Merriwether ( $\nabla$ em3766@nyu.edu )

New York University

Sandra Wittleder

NYU Grossman School of Medicine

Gawon Cho

New York University

Eushavia Bogan

NYU Grossman School of Medicine

Rachel Thomas

New York University

Naja Bostwick

New York University

Binhuan Wang

NYU Grossman School of Medicine

Joseph Ravenell

NYU Grossman School of Medicine

Melanie Jay

NYU Grossman School of Medicine

\section{Research Article}

Keywords: pain, race, racial discrimination, weight, weight discrimination, sex, gender, weight management, obesity

Posted Date: June 28th, 2021

DOl: https://doi.org/10.21203/rs.3.rs-610924/v1 
License: (c) (i) This work is licensed under a Creative Commons Attribution 4.0 International License. Read Full License

Version of Record: A version of this preprint was published at BMC Public Health on December 1st, 2021. See the published version at https://doi.org/10.1186/s12889-021-12199-1. 


\section{Abstract}

Background: Everyday experiences with racial (RD) and weight discrimination (WD) are risk factors for chronic pain in ethnically diverse adults with obesity. However, the individual or combined effects of RD or WD on pain in adults with obesity is not well understood. There are gender differences and sexual dimorphisms in nociception and pain, but the effect of gender on relationships between RD and WD in ethnically diverse adults with obesity has not been examined. The goals of this study were to: 1) examine whether weight and racial discrimination are associated with pain intensity and interference, and 2) explore gender as a moderator of the effects of WD and RD on pain.

Methods: This is a baseline data analysis from a randomized controlled weight-management trial. Eligible participants were English or Spanish-speaking (ages 18-69 years) and had either a body mass index of $\geq 30 \mathrm{~kg} / \mathrm{m}^{2}$ or $\geq 25 \mathrm{~kg} / \mathrm{m}^{2}$ with weight-related comorbidity. WD and RD were measured using the Experiences of Discrimination questionnaire (EOD). Pain outcome (pain interference and intensity) were measured using the PROMIS 29 adult profile V2.1. Linear regression models were performed to determine the associations between WD, RD, and gender, with pain outcomes.

Results. On average participants reported mild pain interference (T-score: $52.65 \pm 10.29)$ and moderate pain intensity $(4.23 \pm 3.15$, scale: $0-10)$. There was a significant interaction effect of RD and gender on pain interference. RD was more strongly associated with pain interference in women $(b=.47, S E=.08, p<$ 001), compared to men $(b=.14, S E=.07, p=.06)$. There were no significant interaction effects between $\mathrm{RD}$ and gender on pain intensity, or between WD and gender on pain interference or intensity.

Conclusions: Pain is highly prevalent in adults with obesity, and is impacted by experiences of racial and weight discrimination. Further, discrimination against adults with obesity and chronic pain could exacerbate existing racial disparities in pain and weight management. Asking ethnically diverse adults with obesity about their pain and their experiences of racial and weight discrimination could help clinicians make culturally informed decisions that address barriers to pain relief and weight loss.

\section{Trial Registration: NCT03006328}

\section{Background}

Discrimination involves negative attitudes or unequal treatment towards individuals based on their characteristics (e.g., race, gender, weight status).(1-3) Research suggests that frequent experiences with discrimination may be a stronger risk factor for pain among racialized groups than their White counterparts,(1) potentially due to Non-Hispanic Black (NHB) and Hispanic/Latino/a/x adults being at risk of multiple forms of discrimination. Particularly NHB and Hispanic/Latino/a/x adults may experience weight-based discrimination in addition to racial discrimination because obesity is highly prevalent among NHB and Latino/a/x adults.(4) Understanding how discrimination may impact pain experiences among individuals with obesity is important given that pain is an established weight-related comorbidity, $(5-7)$ and may further worsen health by functioning as a barrier to weight loss.(8) 
Pain disparities associated with racial discrimination (RD) have been studied extensively. RD has been associated with higher pain intensity,(3) more pain-related disability,(3) more bodily pain,(9) and lower pain tolerance.(10) In addition, weight discrimination (WD) is also a prevalent form of discrimination in the U.S. $(2,11-13)$ and is associated with a wide variety of negative pain-related outcomes, including functional disability, $(14)$ psychiatric comorbidities, $(15)$ weight gain, $(16,17)$ lower health-related quality of life,(18) and higher mortality.(19) Nonetheless, little is known about the impact of WD on the burden of pain, especially among NHB and Hispanic/Latino/a/x adults with obesity, who tend to be underrepresented in pain studies.

Research investigating multiple forms of discrimination suggest that characteristics that are perceived in society as 'modifiable' (e.g. weight) are likely to have a stronger negative effect on psychological health outcomes associated with pain and weight management than discrimination based on 'non-modifiable' factors such as race and ethnicity.(20) Other studies found a dose-response relationship between multiple forms of discrimination and cardiovascular risk,(21) and that WD, but not RD, was significantly associated obesity risk.(19) Based on these findings, it is likely multiple forms of discrimination contribute to the burden of pain among NHB and Hispanic/Latino/a/x individuals. However, studies have not partitioned the effects of racial and weight discrimination on the risk of pain and disability.

Discrimination-related experiences may impact women and men differently.(1, 22) Some evidence suggests that women are more likely to experience negative pain-related health consequences (e.g., psychological stress, anxiety, and pain catastrophizing $)(20,23)$ associated with discrimination, potentially due to the use of maladaptive coping strategies.(24) Female body ideals shared within a society may increase women's risk for encountering WD and exacerbate the negative impact of WD experiences. $(13,25)$ Gender differences in the association between discrimination and pain are understudied in ethnically diverse populations with obesity, in part, due to an underrepresentation of men in weight management research. $(26,27)$

Thus, the primary aim of this study was to examine whether weight and racial discrimination are associated with pain intensity and interference in a racially diverse sample of adults with obesity. $(28,29)$ Our secondary aim was to explore gender as a moderator of the effects of WD and RD on pain. We hypothesized that more experiences with the combination of RD and WD would be associated with higher pain intensity and greater pain interference. The goal of this analysis is to help identify additional pain mechanisms among individuals with obesity.

\section{Methods}

\section{Participants, Study Design, and Setting}

This is an analysis of baseline data from a randomized, controlled clinical trial (RCT) to test the efficacy of technology-assisted health coaching intervention on weight management at two diverse urban healthcare systems in New York City: VA New York Harbor Healthcare System Manhattan Campus (VA) 
and four Montefiore Medical Group (MMG) primary care practices. The four MMG practices - Bronx East, Castle Hill, Grand Concourse, and University Avenue-are affiliated with the New York City Research and Improvement Networking Group. The trial was registered at clinicaltrials.gov (NCT03006328, December $30,2016)$. The sample consisted of 483 primary care patients enrolled in this RCT. Study methods for the clinical trial are published in detail elsewhere.(30) All methods were performed in accordance with the relevant guidelines and regulations approved by the Institutional Review Boards at NYU School of Medicine (\#i16-01445), VA NY Harbor (\#01624), and Albert Einstein College of Medicine in collaboration with the Montefiore Health System (\#2017-7603). All patients gave their informed consent to the study before data collection began. The data were collected between the years 2017 and 2020. At baseline, research assistants conducted in-person visits with patients to assess body measurements (e.g., height and weight) and administer survey measures. Patients received 25 USD in compensation for completing baseline data assessment.

Recruitment. We used queries of electronic health records through the Veterans Health Information Systems and Technology Architecture (VistA), and Clinical Looking Glass ${ }^{T M}$ (CLG), for the VA and MMG, respectively, to identify potentially eligible patients. The majority of patients at the VA identify as male (90\%), compared to $48 \%$ across the MMG clinics. Patient population at both sites are diverse with $21-$ $55 \%$ of patients identifying as Hispanic/Latino/a/ $x$ and $37-53 \%$ as NHB. We sent out invitation letters and followed up with telephone calls to assess patients' interest in participation. If the patient was interested, we performed a phone screen survey and conducted a chart review when necessary to determine eligibility. Patients were then scheduled for the baseline visit.

Eligibility. Participants were primary care patients with either a body mass index (BMI) of $\geq 30 \mathrm{~kg} / \mathrm{m}^{2}$ (obese) or $\geq 25 \mathrm{~kg} / \mathrm{m}^{2}$ (overweight) with weight-related comorbidity (e.g., arthritis, sleep apnea, hypertension). Eligible participants were English or Spanish-speaking primary care patients between the ages of 18-69 years old who had at least one visit with their primary care provider in the past 24 months, access to a telephone, and the ability to travel for in-person visits. Patients with conditions or medications that may affect weight change or impact their ability to participate were excluded. Excluded conditions included metastatic cancer, current chemotherapy or cancer treatment, diabetes, active psychosis, psychoactive substance use, Parkinson's disease, or health problems that may prohibit the patient from participating in walking and/or physical activity such as chest tightness, a heart condition, or severe arthritis. Excluded medications were those for weight-loss and antipsychotic medications.(31) We excluded patients with a history of bariatric surgery or who were being evaluated for bariatric surgery, were pregnant, breastfeeding or planning to become pregnant during the intervention period, or participated in intensive weight management programs ( $>4$ sessions) in the past year. We also excluded patients who did not have self-reported ability to read English or Spanish at the 5th grade level and those with cognitive limitations that prevented them from adequately participating in a weight management program. Additionally, we did not enroll patients who were not interested in losing weight, as well as any patient whose primary care provider stated that they should not participate. 


\section{Measures}

Demographic characteristics. Data collected from the baseline questionnaire included information on participant demographics. Questions contained information on patient gender, civil status, employment, education, race and ethnicity.

Racial Discrimination. Participants completed the Experiences of Discrimination questionnaire (EOD),(32) which has been studied in patients with obesity and chronic pain. $(10,21,33,34)$ To assess experiences of discrimination due to race or ethnicity, patients were asked, "Have you ever experienced discrimination, been prevented from doing something, or been hassled or made to feel inferior in any of the following situations because of your race, ethnicity or color?" The frequency of nine discrimination experiences was assessed over a patient's lifetime: "at school"; "getting hired"; "at work"; "getting housing"; "getting medical care"; "getting service at a store or restaurant"; "getting credit", "bank loans or a mortgage"; "on the street or in a public place"; and "from the police or courts". Available answer choices included: never (0), yes, once (1), yes, 2-3 times (2.5), and yes, 4-5 times or more (5). (Cronbach's Alpha $=.84)$. We combined answers to a frequency sum score ranging from 0 to 45 . Higher scores reflect more experiences with racial discrimination.

Weight Discrimination. We assess experienced discrimination due to weight similar to previous studies, which included diverse representative samples.(15, 21, 25, 35) Participants were administered a question from Wave 2 of the National Epidemiologic Survey on Alcohol and Related Conditions (NESARC), which were based on the EOD survey.(32) Participants answered the question, "In the last 12 months, how often did you experience discrimination because of your weight?". The frequency of five scenarios was examined: "in your ability to obtain healthcare or health insurance coverage"; "in the way you were treated when you receive care"; "in public settings, like on the street", "in restaurants or stores or on public transportation like buses or airplanes"; "in obtaining a job, or getting admitted to a school or training program"; and "in any other situation, like in the courts or by the police or when obtaining housing". The response options were Almost never (1), Sometimes (2), Fairly Often (3), and Very Often (4). If participants responded Sometimes, Fairly often, or Very Often to any of the questions, we categorized them as reporting weight discrimination. We created a dichotomous variable coding: no reported weightbased discrimination (0) and reported weight-based discrimination (1) for each patient (Cronbach's Alpha $=.65$ ). This categorization approach is also consistent with previous studies.(36)

Pain measurement. The pain interference and pain intensity scales of the Patient-Reported Outcomes Measurement Information System (PROMIS) 29 adult profile V2.1 were used to measure chronic pain. The PROMIS-29 has been widely used including in populations with obesity and chronic pain.(37-43) Participants answered four questions addressing pain interference (Cronbach's Alpha $=.94)$ : "In the past 7 days, how much did pain interfere with your day to day activities", "work around the house", "your ability to participate in social activities", "with your household chores". Responses were on a scale from one to five, and defined as: not at all (1), a little bit (2), somewhat (3), quite a bit (4), very much (5). We combined answers to a raw sum score with a minimum of 4 and a maximum of 20 . For each participant, we 
translated the total raw score into a T-score, which has a mean of 50 and a standard deviation $(S D)$ of 10 , based on calibration testing performed on a large sample of the general population.(44) A person with a T-score of 40 is, therefore, one $S D$ below the average for the United States general population.

Additionally, participants rated their pain intensity ("How would you rate your pain on average?") on a scale from no pain(0) to extreme pain(10).

BMI. Participants' BMI was calculated by dividing their weight in kilograms $(\mathrm{kg})$ by the square of their height in meters $(\mathrm{m})$, expressed as $\mathrm{kg} / \mathrm{m}^{2}$. Body weight and height were collected at baseline. The patients' height was assessed once using the SECA 213 Portable Height, and the measurements were rounded up to the nearest 0.50 centimeter $(\mathrm{cm})$. Patients were asked to modify hairstyles and remove their shoes as well as any extraneous clothing, if possible. Baseline height was measured twice, and the average was used for analysis. Patient weight measurements were obtained via the HealthOMeter 349KLX Digital Medical Weight Scale using a standardized protocol, which included weighing the patients twice without shoes or heavy garments. Weight was measured twice to the nearest 0.10 pound (lbs.). If the first two weights differed by $0.50 \mathrm{lbs}$. or more, we repeated the measurement once more and took the average of the two measures closest in value.

\section{Statistical Analysis}

Demographic characteristics were summarized using descriptive statistics, with mean and standard deviation for continuous variables or frequencies and percentages for categorical variables. Differences in pain scores and RD scores by demographic characteristics were compared by the Mann-Whitney U test for dichotomous variables, and the Kruskal-Wallis test for categorical variables with more than two levels. Pairwise comparisons were done using Bonferroni correction. Differences in WD by demographic characteristics were compared using the Chi-squared test. Associations between pain scores and race or ethnic discrimination scores were quantified by Spearman's rho correlation coefficient $\left(r_{\mathrm{s}}\right)$. Differences in pain scores by weight discrimination group were compared by the Mann-Whitney $\mathrm{U}$ test. We used simultaneous linear regression models to examine independent relationships between WD, RD, and gender and pain intensity and pain interference. We also analyzed the interaction effects of WD, RD, and gender on pain intensity and interference. We chose this model because we have no theoretical basis for considering any variable to be prior to any other. We added $\mathrm{BMI}$, age, and race as confounding variables because they have been shown to be related to discrimination measures (independent variables) $(45,46)$ and pain measures (dependent variables) $(47,48)$ in previous research. Race was classified into four categories using indicator variables: Hispanic, Non-Hispanic White (reference group), Non-Hispanic Black, and Non-Hispanic Other. In our sample, enrollment site was associated with both RD and pain interference score and was, hence, also entered as a covariate (Table 1). We entered all main effects and 2- and 3- way interactions among WD, RD, and gender as predictors, with BMI, age and enrollment site entered as covariates. If an interaction effect was significant (at the $p>.05$ level), lower-order interactions and main effects nested beneath were not interpreted. Significant interactions were probed and plotted with the PROCESS macro for SPSS (version 3.04). A two-sided p-value $<0.05$ was considered to be 
statistically significant, and all analyses were conducted using IBM SPSS Statistics for Windows, Version 25.0 (Armonk, NY: IBM Corp).

\section{Results}

\section{Participant Characteristics}

Our sample was balanced in gender ( $56.3 \%$ female; $43.7 \%$ male) and the average age was 49.66 year $(S D=12.07)$. The average BMI was $34.83 \mathrm{~kg} / \mathrm{m} 2(S D=6.18)$, which falls in the obese range $(B M I \geq 30)$. Almost half of all participants identified as NHB (43.7\%) and a little less than half of the participants (40.8\%) reported their ethnicity as Hispanic/Latino/a/x. Pain interference had an average T-value of 52.65 $(S D=10.29)$. Pain intensity was 4.23 on average $(S D=3.15)$, and $59.1 \%$ of participants reported a pain intensity of 4 or higher on a scale from 0 to 10 . Most participants worked full-time or part-time $(62.7 \%)$. Approximately one-third (33.6\%) of participants graduated from a 4-year college with $10 \%$ having earned a professional or graduate degree. Almost half (39.3\%) of all patients were single/never married, and a similar number (36.2\%) were married or in a marriage-like relationship (Table 1).

\section{Differences in Pain Interference and Pain Intensity}

Participants enrolled at the VA NY Harbor site reported higher pain interference than participants enrolled at MMG clinics. Patients who were unemployed or looking for work reported higher pain interference than patients who worked full-time. Retired patients experienced greater pain interference compared to patients who worked full or part-time. We did not observe differences in pain scores between gender, racial/ethnic groups, or marital status (Table 1).

\section{Differences in Race and Weight-Based Discrimination}

Participants enrolled in at the VA NY Harbor site reported more instances of RD than those enrolled at MMG clinics. Men-identifying participants' average RD scores that were 1.52 times greater than womenidentifying participants. Further, NHB had RD scores that were greater than 2.0 times the average score reported by both Non-Hispanic White and Hispanic/Latino/a/x individuals. We did not observe differences in gender, racial/ethnic groups, employment, education, and between those who reported WD and those who did not report WD (Table 1). 
Table 1 Participant Characteristics in Pain Outcomes (Intensity and Interference), Racial Discrimination (RD), and Weight Discrimination (WD)

$\begin{array}{lll}\text { Total } & \begin{array}{l}\text { Pain } \\ \text { Intensity }\end{array} & \begin{array}{l}\text { Pain Inter- } \\ \text { ference }\end{array}\end{array}$

No WD

RD WD

\section{Enrollment Site}

$\begin{array}{lllllll}\text { MMG } & 241 & 4.00 & \mathbf{5 0 . 9 8} & \mathbf{4 . 9 1} & 205 & 36 \\ & (49.9 \%) & (3.30)^{1} & (\mathbf{9 . 8 4})^{1 *} & (\mathbf{6 . 5 3})^{1 *} & (85.1 \%)^{4} & (14.9 \%)^{4} \\ \text { VA } & 242 & 4.48 & \mathbf{5 4 . 3 5} & \mathbf{9 . 3 2} & 194 & 48 \\ & (50.1 \%) & (2.97)^{1} & (\mathbf{( 1 0 . 4 6 )})^{\mathbf{1 *}} & \mathbf{( 9 . 9 3 ) ^ { 1 * }} & (80.1 \%)^{4} & (19.9 \%)^{4}\end{array}$

\section{Gender}

$\begin{array}{lllllll}\text { Women } & 273 & 4.21 & 52.11 & \mathbf{5 . 7 9} & 224 & 48 \\ & (56.4 \%) & (3.22)^{1} & (10.26)^{1} & \left(\mathbf{7 . 5 4 ) ^ { 1 * }}\right. & (82.3 \%)^{4} & (17.7 \%)^{4} \\ \text { Men } & 211 & 4.28 & 53.40 & \mathbf{8 . 8 1} & 175 & 36 \\ & (43.6 \%) & (3.05)^{1} & (10.29)^{1} & (\mathbf{9 . 7 1})^{1 *} & (82.9 \%)^{4} & (17.1 \%)^{4}\end{array}$

\section{Race/Ethnicity}

\begin{tabular}{|c|c|c|c|c|c|c|}
\hline Non-Hispanic Black & $\begin{array}{l}211 \\
(43.6 \%)\end{array}$ & $\begin{array}{l}4.17 \\
(3.17)^{2}\end{array}$ & $\begin{array}{l}53.03 \\
(10.44)^{2}\end{array}$ & $\begin{array}{l}10.26 \\
(10.07)^{2, a, b}\end{array}$ & $\begin{array}{l}169 \\
(80.5 \%)^{4}\end{array}$ & $\begin{array}{l}41 \\
(19.5 \%)^{4}\end{array}$ \\
\hline Non-Hispanic White & $\begin{array}{l}48 \\
(9.9 \%)\end{array}$ & $\begin{array}{l}4.08 \\
(2.77)^{2}\end{array}$ & $\begin{array}{l}53.10 \\
(10.83)^{2}\end{array}$ & $\begin{array}{l}3.83 \\
(5.66)^{2, a}\end{array}$ & $\begin{array}{l}39 \\
(81.2 \%)^{4}\end{array}$ & $\begin{array}{l}9 \\
(18.8 \%)^{4}\end{array}$ \\
\hline Non-Hispanic Other & $\begin{array}{l}26 \\
(5.4 \%)\end{array}$ & $\begin{array}{l}3.56 \\
(3.03)^{2}\end{array}$ & $\begin{array}{l}51.27 \\
(12.63)^{2}\end{array}$ & $\begin{array}{l}6.76 \\
(8.29)^{2}\end{array}$ & $\begin{array}{l}22 \\
(84.0 \%)^{4}\end{array}$ & $\begin{array}{l}4 \\
(16.0 \%)^{4}\end{array}$ \\
\hline Hispanic/Latino/a/x & $\begin{array}{l}197 \\
(40.7 \%)\end{array}$ & $\begin{array}{l}4.45 \\
(3.21)^{2}\end{array}$ & $\begin{array}{l}52.39 \\
(9.71)^{2}\end{array}$ & $\begin{array}{l}4.66 \\
(6.44)^{2, b}\end{array}$ & $\begin{array}{l}167 \\
(84.8 \%)^{4}\end{array}$ & $\begin{array}{l}30 \\
(15.2 \%)^{4}\end{array}$ \\
\hline
\end{tabular}

\section{Employment Status}

\begin{tabular}{|c|c|c|c|c|c|c|}
\hline Working full-time & $\begin{array}{l}247 \\
(51.0 \%)\end{array}$ & $\begin{array}{l}3.92 \\
(3.13)^{2}\end{array}$ & $\begin{array}{l}51.05 \\
(9.55)^{2, a, b}\end{array}$ & $\begin{array}{l}6.62 \\
(7.95)^{2}\end{array}$ & $\begin{array}{l}206 \\
(83.7 \%)^{4}\end{array}$ & $\begin{array}{l}40 \\
(16.3 \%)^{4}\end{array}$ \\
\hline Working part-time & $\begin{array}{l}56 \\
(11.6 \%)\end{array}$ & $\begin{array}{l}3.95 \\
(3.30)^{2}\end{array}$ & $\begin{array}{l}50.19 \\
(9.42)^{2, c}\end{array}$ & $\begin{array}{l}4.49 \\
(6.02)^{2}\end{array}$ & $\begin{array}{l}51 \\
(91.1 \%)^{4}\end{array}$ & $5(8.9 \%)^{4}$ \\
\hline $\begin{array}{l}\text { Unemployed or laid } \\
\text { off/Looking for work }\end{array}$ & $\begin{array}{l}62 \\
(12.7 \%)\end{array}$ & $\begin{array}{l}4.92 \\
(2.93)^{2}\end{array}$ & $\begin{array}{l}55.41 \\
(10.30)^{2, b}\end{array}$ & $\begin{array}{l}8.83 \\
(10.14)^{2}\end{array}$ & $\begin{array}{l}46 \\
(74.8 \%)^{4}\end{array}$ & $\begin{array}{l}16 \\
(25.2 \%)^{4}\end{array}$ \\
\hline Student & $\begin{array}{l}15 \\
(3.1 \%)\end{array}$ & $\begin{array}{l}3.37 \\
(2.91)^{2}\end{array}$ & $\begin{array}{l}53.59 \\
(10.74)^{2}\end{array}$ & $\begin{array}{l}5.23 \\
(6.96)^{2}\end{array}$ & $\begin{array}{l}12 \\
(80.0 \%)^{4}\end{array}$ & $\begin{array}{l}3 \\
(20.0 \%)^{4}\end{array}$ \\
\hline
\end{tabular}




\begin{tabular}{|c|c|c|c|c|c|c|}
\hline $\begin{array}{l}\text { Keeping house or } \\
\text { aising children full time }\end{array}$ & $\begin{array}{l}13 \\
(2.7 \%)\end{array}$ & $\begin{array}{l}4.23 \\
(4.02)^{2}\end{array}$ & $\begin{array}{l}51.60 \\
(10.85)^{2}\end{array}$ & $\begin{array}{l}6.54 \\
(9.66)^{2}\end{array}$ & $\begin{array}{l}10 \\
(76.9 \%)^{4}\end{array}$ & $\begin{array}{l}3 \\
(23.1 \%)^{4}\end{array}$ \\
\hline Ret & $\begin{array}{l}91 \\
(18.8 \%)\end{array}$ & $\begin{array}{l}4.91 \\
(3.02)^{2}\end{array}$ & $\begin{array}{l}56.77 \\
(11.20)^{2, a, c}\end{array}$ & $\begin{array}{l}9.35 \\
(10.42)^{2}\end{array}$ & $\begin{array}{l}74 \\
(81.3 \%)^{4}\end{array}$ & $\begin{array}{l}17 \\
(18.7 \%)^{4}\end{array}$ \\
\hline
\end{tabular}

\section{Education}

\begin{tabular}{|c|c|c|c|c|c|c|}
\hline Grades 5 through 11 & $\begin{array}{l}20 \\
(4.0 \%)\end{array}$ & $\begin{array}{l}4.70 \\
(3.67)^{2}\end{array}$ & $\begin{array}{l}51.48 \\
(9.96)^{2}\end{array}$ & $\begin{array}{l}3.15 \\
(5.72)^{2}\end{array}$ & $\begin{array}{l}16 \\
(80.0 \%)^{4}\end{array}$ & $\begin{array}{l}4 \\
(20.0 \%)^{4}\end{array}$ \\
\hline Grade 12 or GED & $\begin{array}{l}112 \\
(22.9 \%)\end{array}$ & $\begin{array}{l}4.57 \\
(3.38)^{2}\end{array}$ & $\begin{array}{l}52.93 \\
(10.38)^{2}\end{array}$ & $\begin{array}{l}6.26 \\
(9.35)^{2}\end{array}$ & $\begin{array}{l}89 \\
(80.2 \%)^{4}\end{array}$ & $\begin{array}{l}22 \\
(19.8 \%)^{4}\end{array}$ \\
\hline Associates degree & $\begin{array}{l}58 \\
(11.9 \%)\end{array}$ & $\begin{array}{l}3.07 \\
(2.99)^{2, a}\end{array}$ & $\begin{array}{l}51.14 \\
(10.76)^{2}\end{array}$ & $\begin{array}{l}6.81 \\
(6.86)^{2}\end{array}$ & $\begin{array}{l}52 \\
(89.7 \%)^{4}\end{array}$ & $\begin{array}{l}6 \\
(10.3 \%)^{4}\end{array}$ \\
\hline Some college & $\begin{array}{l}133 \\
(27.2 \%)\end{array}$ & $\begin{array}{l}4.69 \\
(3.12)^{2, a}\end{array}$ & $\begin{array}{l}53.39 \\
(10.42)^{2}\end{array}$ & $\begin{array}{l}7.20 \\
(8.03)^{2}\end{array}$ & $\begin{array}{l}109 \\
(83.7 \%)^{4}\end{array}$ & $\begin{array}{l}21 \\
(16.3 \%)^{4}\end{array}$ \\
\hline College 4 years & $\begin{array}{l}102 \\
(20.9 \%)\end{array}$ & $\begin{array}{l}3.89 \\
(2.85)^{2}\end{array}$ & $\begin{array}{l}52.72 \\
(9.77)^{2}\end{array}$ & $\begin{array}{l}7.65 \\
(9.29)^{2}\end{array}$ & $\begin{array}{l}83 \\
(83.0 \%)^{4}\end{array}$ & $\begin{array}{l}17 \\
(17.0 \%)^{4}\end{array}$ \\
\hline $\begin{array}{l}\text { Some graduate or } \\
\text { professional training }\end{array}$ & $\begin{array}{l}12 \\
(2.5 \%)\end{array}$ & $\begin{array}{l}4.25 \\
(3.08)^{2}\end{array}$ & $\begin{array}{l}53.51 \\
(11.79)^{2}\end{array}$ & $\begin{array}{l}10.88 \\
(9.89)^{2}\end{array}$ & $\begin{array}{l}7 \\
(58.3 \%)^{4}\end{array}$ & $\begin{array}{l}5 \\
(41.7 \%)^{4}\end{array}$ \\
\hline $\begin{array}{l}\text { Graduate or } \\
\text { Professional degree }\end{array}$ & $\begin{array}{l}50 \\
(10.2 \%)\end{array}$ & $\begin{array}{l}4.28 \\
(2.91)^{2}\end{array}$ & $\begin{array}{l}52.25 \\
(10.31)^{2}\end{array}$ & $\begin{array}{l}8.63 \\
(9.74)^{2}\end{array}$ & $\begin{array}{l}41 \\
(82.0 \%)^{4}\end{array}$ & $\begin{array}{l}9 \\
(18.0 \%)^{4}\end{array}$ \\
\hline \multicolumn{7}{|l|}{ Marital Status } \\
\hline Single/Never Married & $\begin{array}{l}190 \\
(39.3 \%)\end{array}$ & $\begin{array}{l}3.87 \\
(3.23)^{2}\end{array}$ & $\begin{array}{l}52.18 \\
(10.70)^{2}\end{array}$ & $\begin{array}{l}7.11 \\
(7.83)^{2}\end{array}$ & $\begin{array}{l}153 \\
(80.5 \%)^{4}\end{array}$ & $\begin{array}{l}37 \\
(19.5 \%)^{4}\end{array}$ \\
\hline $\begin{array}{l}\text { Married or marriage- } \\
\text { like relationship }\end{array}$ & $\begin{array}{l}175 \\
(36.2 \%)\end{array}$ & $\begin{array}{l}4.51 \\
(3.09)^{2}\end{array}$ & $\begin{array}{l}53.01 \\
(9.85)^{2}\end{array}$ & $\begin{array}{l}6.81 \\
(9.38)^{2}\end{array}$ & $\begin{array}{l}152 \\
(86.9 \%)^{4}\end{array}$ & $\begin{array}{l}23 \\
(13.1 \%)^{4}\end{array}$ \\
\hline Separated & $\begin{array}{l}23 \\
(4.6 \%)\end{array}$ & $\begin{array}{l}4.14 \\
(3.09)^{2}\end{array}$ & $\begin{array}{l}51.45 \\
(10.10)^{2}\end{array}$ & $\begin{array}{l}5.93 \\
(8.29)^{2}\end{array}$ & $\begin{array}{l}18 \\
(77.3 \%)^{4}\end{array}$ & $\begin{array}{l}5 \\
(22.7 \%)^{4}\end{array}$ \\
\hline Divorced & $\begin{array}{l}82 \\
(17.0 \%)\end{array}$ & $\begin{array}{l}4.23 \\
(3.08)^{2}\end{array}$ & $\begin{array}{l}52.38 \\
(10.02)^{2}\end{array}$ & $\begin{array}{l}7.42 \\
(8.74)^{2}\end{array}$ & $\begin{array}{l}66 \\
(81.5 \%)^{4}\end{array}$ & $\begin{array}{l}15 \\
(18.5 \%)^{4}\end{array}$ \\
\hline Widowed & $\begin{array}{l}13 \\
(2.7 \%)\end{array}$ & $\begin{array}{l}6.00 \\
(2.65)^{2}\end{array}$ & $\begin{array}{l}58.57 \\
(11.32)^{2}\end{array}$ & $\begin{array}{l}10.62 \\
(11.21)^{2}\end{array}$ & $\begin{array}{l}9 \\
(69.2 \%)^{4}\end{array}$ & $\begin{array}{l}4 \\
(30.8 \%)^{4}\end{array}$ \\
\hline
\end{tabular}

Note. $\mathrm{RD}=$ racial discrimination, $\mathrm{WD}=$ weight discrimination

Hispanic/Latino/a/x of any race including Black $(n=25)$, White/Caucasian $(n=26)$, None $(n=137)$; Multiple $(n=8)$, and Tainos Indian $(n=1)$. All other race categories are assumed to be non-Hispanic; Other include Asian ( $n=5)$; American Indian/Alaskan Native $(n=1)$, Native-Hawaiian/Pacific Islander $(n=$ $2)$, Multiple $(n=9)$, None $(n=6)$, West Indian $(n=2)$, and Middle Eastern $(n=1)$. 
${ }^{1}$ Mann-Whitney U tests, ${ }^{2}$ Kruskal-Wallis tests, ${ }^{4}$ Chi-Square test.

${ }^{*} \mathrm{p}<.05$ (two-tailed). Pairwise comparisons used Bonferroni correction. Means sharing a common subscript $(\mathrm{a}, \mathrm{a}, \mathrm{a})$ are statistically different at $p>.05$.

\section{Aim 1: Characterize association between RD, WD, and pain.}

$\mathrm{RD}$ was positively correlated with greater pain interference, $r_{\mathrm{s}}=.27, p<.001$, and higher pain intensity scores, $r_{\mathrm{s}}=.16, p=.001$. Similarly, patients who experienced WD reported greater pain interference, $(M=57.18, S D=9.98$ vs. $M=51.75, S D=10.11), Z=-4.43, p<.001$, and higher pain intensity scores, $(M=5.29$, $S D=2.78$ vs. $M=4.03, S D=3.17), Z=-3.41, p=.001$, compared with those who did not report WD. After adjusting for $\mathrm{BMI}$, age, and enrollment site, findings from the linear regression analysis showed that only RD predicted greater pain interference (Table 2), and higher pain intensity (Table 3). Interestingly, there was no significant interaction effect between RD and WD on pain outcomes (Tables $2 \& 3$ ).

\section{Aim 2: Explore gender as a moderator of the association between RD, WD, and pain interference and intensity.}

Linear regression analysis revealed a significant interaction effect of RD X Gender on pain interference (Table 2). Among woman-identifying participants, RD was more strongly associated with pain interference $b=.47, S E=.08, \mathrm{Cl}[.303$ to .629$]$, compared to the association among men-identifying participants, $b=.14, S E=.07, \mathrm{CI}[-.005$ to .282$]$ (Table 2, Figure 1a). There were also a significant interaction effect between RD X Gender on pain intensity (Table 3). RD was associated with pain intensity among women-identifying participants but not in men-identifying participants, $b=.08, S E=.03, p=.00195 \%$ $\mathrm{Cl}[.033$ to .136$]$, but not in men, $b=.03, S E=.02, p=2995 \% \mathrm{Cl}[-.021$ to .070$]$ (Figure 1b). The interactions between WD X Gender on pain interference or pain intensity were not significant (Tables $2 \& 3$ ).

Table 2 Pain Interference

\begin{tabular}{|lllllllll|}
\hline & Standardized Beta & SE & $t$ & $p$ & $F$ & $d f$ & $p$ & Adj. R2 \\
\hline Model & & & & & 6.01 & 12 & $<.001^{*}$ & 0.11 \\
\hline Gender & 0.06 & 1.39 & 0.96 & 0.34 & & & & \\
\hline WD $^{f}$ & 0.10 & 2.11 & 1.25 & 0.21 & & & & \\
\hline RD & $\mathbf{0 . 4 6}$ & $\mathbf{0 . 1 1}$ & $\mathbf{4 . 8 9}$ & $<.001^{*}$ & & & & \\
\hline RDxWD & -0.14 & 0.12 & -1.61 & 0.11 & & & & \\
\hline RDxGender & -0.30 & $\mathbf{0 . 1 2}$ & -3.30 & $\mathbf{0 . 0 0 1 *}$ & & & \\
\hline WDxGender & 0.07 & 2.72 & 1.09 & 0.28 & & & & \\
\hline
\end{tabular}

Note. 
${ }^{\mathrm{a}} 0=$ female, $1=$ male, ${ }^{\mathrm{b}} 0=\mathrm{VA}, 1=\mathrm{MMG},{ }^{\mathrm{c}} 0=$ Non-Hispanic White, $1=$ Non-Hispanic Black, ${ }^{\mathrm{d}} 0=$ White, $1=$ Hispanic $/$ Latino $/ a / x,{ }^{e} 0=$ White, $1=$ Non-Hispanic Other, ${ }^{f} 0=$ no WD, $1=$ WD.

${ }^{\star} p<.05$

Table 3 Pain Intensity

\begin{tabular}{|llllllllll|}
\hline & Standardized Beta & SE & $t$ & $p$ & $F$ & $d f$ & $p$ & Adj. R2 \\
\hline Model & & & & & & & & \\
\hline Gender $^{\mathrm{a}}$ & 0.01 & & & & 2.95 & 12 & $.001^{*}$ & 0.05 \\
\hline WD $^{f}$ & 0.06 & 0.44 & 0.15 & 0.88 & & & & \\
\hline RD & $\mathbf{0 . 2 9}$ & 0.67 & 0.73 & 0.47 & & & & \\
\hline RDxWD & -0.10 & $\mathbf{0 . 0 4}$ & $\mathbf{2 . 1 0}$ & $\mathbf{0 . 0 0 4 ^ { * }}$ & & & & \\
\hline RDxGender & $-\mathbf{- 0 . 2 1}$ & 0.04 & -1.06 & 0.29 & & & \\
\hline WDxGender & 0.10 & $\mathbf{0 . 0 4}$ & $-\mathbf{- 2 . 2 0}$ & $\mathbf{0 . 0 3 *}$ & & & & \\
\hline
\end{tabular}

Note. ${ }^{\mathrm{a}} 0=$ female, $1=$ male, ${ }^{\mathrm{b}} 0=\mathrm{VA}, 1=\mathrm{MMG},{ }^{\mathrm{c}} 0=$ Non-Hispanic White, $1=$ Non-Hispanic Black, ${ }^{\mathrm{d}} 0=$ White, $1=$ Hispanic/Latino/a/ $x,{ }^{e} 0=$ White, $1=$ Non-Hispanic Other, ${ }^{f} 0=$ no WD, $1=$ WD.

Age, race, and BMI were entered as covariates. For complete model statistics, see supplemental materials.

${ }^{*} p<.05$

\section{Discussion}

The current study characterized the relationships between experiences with two types of discrimination (racial, weight) and pain outcomes, and investigated if gender moderated these associations. Results from this study confirm a high prevalence (59.1\%) of moderate pain (4 out of 10 or higher) and pain interference in adults with obesity (Mean t-score $=52.65$ ). Also, experiences with racial discrimination (RD) are significant predictors of pain intensity and pain interference in well represented sample of NHB and Hispanic/Latino/a/x adults with obesity having various chronic pain conditions. Further, the association between pain interference and racial discrimination is moderated by gender identity. These results suggest that in an ethnically diverse sample of adults with obesity, men report more frequent experiences with racial discrimination. However, the association between the frequency of experiences with racial discrimination and pain interference is stronger in women. Another key finding is that participants who reported to have experienced weight discrimination had significantly greater pain 
interference, higher pain intensity, more experiences with racial discrimination, and higher BMI. However, weight discrimination was not a significant predictor of pain intensity or pain interference after statistical adjustment for experiences with racial discrimination, age, and BMI. From these findings, we can infer that weight and racial discrimination are experientially distinct phenomena, and thus, have a differential impact on the pain experience in adults with obesity. Surprisingly, there were no racial or gender differences in pain intensity or pain interference. To our knowledge, this is the first investigation that investigated gender-based differences in the relationships between racial and weight discrimination on pain in a large sample of NHB and Hispanic/Latino/a/x adults with obesity.

Adults with obesity have a disproportionate burden of chronic pain, and NHB and Approximately $75 \%$ of adults with obesity have chronic pain compared with $20.4 \%$ of the U.S. population.(49) Recent data in the United States have also shown that the age-adjusted prevalence of chronic pain is higher in women and military veterans.(49) Results from this study show a high prevalence of self-reported pain in a diverse sample of participants in a behavioral weight loss program, consistent with previous studies.(50) While $\mathrm{NHB}$ and Hispanic/Latino/a/x adults are known to have a disproportionately higher obesity prevalence, (51) there were no significant differences in pain intensity or pain interference between racial groups in our study population. This contrasts with previous findings of higher self-reported pain in NHB, Hispanic/Latino/a/x, and Asian compared with NHW participants (cite), though the results are inconsistent. Chronic pain prevalence is higher in NHB adults compared with NHW adults(52) in experimental $(53,54)$ and clinical $(55,56)$ settings. Further, Hispanic/Latino/a/x adults, particularly older adults, tend to have lower pain ratings and report less interference with functional activities compared with NHB and NHW adults.(57-59) Zettel-Watson et al. showed that $60 \%$ of older Mexican-American adults reported pain at multiple body sites, moderate to severe pain intensity, and that pain interfered with their normal work over the past four weeks.(57) Importantly, although pain is associated with health outcomes that are critical to the success of behavioral weight loss programs,(50) it is often unaddressed in weight management.(60) Our results highlight the need to query the magnitude and impact of pain so that pain interventions could be successfully incorporated into a weight management program. Moreover, given the underrepresentation of NHB and Hispanic/Latino/a/x individuals with obesity in pain studies, our findings suggest that experiences of discrimination specific to race/ethnicity and weight are salient features of the pain experience in this population that warrant further investigation.

Experiences with racial discrimination on NHB adults have deleterious effects on pain, obesity, and other health outcomes. $(1,9,10,23,34,59,61-63)$ However, experiences with racial discrimination in Hispanic/Latino/a/x and other racialized groups are not well described. NHB adults in the current study reported more experiences of racial discrimination than Non-Hispanic White adults, $(1,9,10,23,59,63)$ and more frequent experiences with racial discrimination were significantly associated with a higher pain intensity and more pain interference after adjusting for confounding variables. Altered nociceptive processing (e.g., heat pain tolerance), psychological factors, and sex/gender differences have been implicated as possible mechanisms underlying the relationship between racial discrimination and pain in NHB adults. $(23,33,53,64-66)$ Our findings that NHB study participants reported more experiences of racial discrimination than Hispanic/Latino/a/x participants. These results suggest that NHB and 
Hispanic/Latino/a/s groups have different experiences with racial discrimination that may influence how discrimination based on race affect pain responses.(57) A potential reason for the differences in the reported instances of racial discrimination between NHB and Hispanic/Latino/a/x adults with obesity in the current study could be that Hispanic/Latino/a/x adults are not often specifically asked about the salient features of their experiences with racial discrimination such as language concordance, level of acculturation, and immigration status.(67) In previous studies, Hispanic/Latino/a/x adults have been asked about their experiences with racial or ethnic discrimination and its impact on pain in the context of access to primary care,(68) provider bias,(68) patient-provider language discordance, and immigration status.(69) Level of acculturation and assimilation into the dominant culture have also been cited as mechanisms of discrimination by providers in a sample of Mexican-Americans.(70) Furthermore, the omnipresent fear of deportation - regardless of citizenship status - is significantly associated with painrelated outcomes, specifically stress and depression, as well as missed appointments for pain treatment. (69) These findings suggest a limitation in the way that questions about experiences with discrimination are asked to Hispanic/Latino/a/x adults. Thus, it is prudent to employ multimodal approaches to the examination of the impact of racial discrimination on pain in NHB and Hispanic/Latino/a/x adults with obesity. Moreover, one might consider asking specific questions related to fear of deportation and level of acculturation to ethnically diverse adults with obesity and chronic pain that have an immigrant experience.

Although sex and gender differences in the prevalence and trajectory of select chronic pain conditions have been well established,(71-82) studies reporting gender differences in the relationships between racial discrimination and pain outcomes in ethnically diverse pain populations have been sparse and inconclusive. In a robust sample of primary care patients with chronic musculoskeletal pain, women reported greater pain interference than men.(83) The majority of the female cohort (54\%) were NHB women. Terry et al. found that despite reporting more experiences with racial discrimination, there were no significant relationships to pain in older NHB men with knee osteoarthritis.(23) Conversely, in a sample of older NHB men (military veterans), racial discrimination was a significant predictor of bodily pain.(9) Notably, NHB and Hispanic/Latino/a/x men with obesity are frequently underrepresented in pain studies, so sex and gender differences in pain interference and experiences with racial discrimination are particularly not well understood in these patient populations. The current study shows that the association between racial discrimination, pain interference, and pain intensity is stronger is womenidentifying participants with obesity whom identify as NHB or Hispanic/Latino/a/x. Previous findings from a large, multi-ethnic cohort show significant relationships between experiences with racial discrimination and bodily pain in Japanese, Chinese, African American, Caucasian, and Hispanic women, but they did not compare their experiences to men.(59) In the same study, NHB women also reported having more frequent experiences with racial discrimination whereas Hispanic/Latino/a/x women reported the lowest frequency.(59) Conversely, Hispanic/Latino/a/x women had the highest pain ratings at baseline compared with NHB, NHW, Japanese, Chinese women (52). Dugan et al. posited that other forms of discrimination, particularly related to gender and English fluency, could have also been captured by the EOD in their study cohort though not directly assessed.(59) 
Some purported mechanisms for the gender differences in the relationships between experiences with racial discrimination and pain interference are related to differences in affective dimensions of pain such as coping, pain self-efficacy, and pain beliefs.(23, 71, 72, 84-92) Pain catastrophizing, a cluster of negative emotions related to magnification, rumination, and helplessness around pain,(93) and perceived stress have been found to moderate the association between discrimination, pain intensity, and pain interference in women when demographic variables are controlled.(23) Although stress was not measured in this investigation, women participants may have experienced stress more intensely than their male counterparts which could explain greater pain interference reported from women despite reporting less discrimination than men.(23) Surprisingly, there were no race or gender differences in pain interference in our sample population. However, the impact of adiposity and body image are under recognized forms of discrimination that may influence the chronicity and management of chronic pain, and should be assessed in pain and weight management.

Weight discrimination is increasingly recognized as a social determinant of health. Racial and weight discrimination have been identified as the most common forms of repeated daily forms of discrimination in racially and ethnically diverse populations of adults with obesity.(94) Importantly, weight discrimination is associated with increases in $\mathrm{BMI}$, and weight gain.(16) The current study shows that participants who identify as NHB or Hispanic/Latino/a/x were able to disentangle their experiences with weight discrimination from their experiences with racial discrimination. Gee et al. reported similar findings in a large cohort of Asian ethnic groups in the context of increased BMI.(95) The group found that weight discrimination was significantly associated with increased BMI. Further, the associations between racial discrimination and BMI were significant when controlling for the influence of weight discrimination. Of note, the majority of participants in the sample were not classified as having obesity using World Health Organization (WHO) criteria $(<10 \%)$. Other researchers have reported that NHB participants cited body appearance, in addition to racial discrimination, as a potential reason for their experiences with discrimination. $(1,2)$ In the current study, weight discrimination was not associated with pain outcomes after statistical adjustment though participants that reported having experiences with weight discrimination had significantly higher pain intensity, greater pain interference, and more experiences with racial discrimination. A potential reason for these discrepant findings is that the number of participants reporting experiences with weight discrimination were underrepresented in the total study population (< $25 \%$ ). Thus, we may have been underpowered to analyze the contribution of weight discrimination to the variance in pain intensity and interference. Mehok and colleagues suggest that patients' weight and gender identity influenced observers' perceptions of pain severity, the rate of referral for physical therapy services, and recommendations to engage in physical activity as an adjuvant therapy for pain control. (96)

There were some limitations associated with the study. First, this is a secondary data analysis; study participants were not recruited based on the presence or absence of a regional or widespread musculoskeletal pain condition. However, our findings may be more generalizable to adult populations with obesity. Secondly, we did not include clinical or laboratory-based assessments of other 
biopsychosocial aspects of chronic pain. Lastly, we did not assess anticipatory or enacted discrimination which could have different relationships to pain intensity or pain interference.

In summary, the current study found that pain is prevalent in adults with obesity participating in a comprehensive behavioral weight management program. Further, we have expanded on results from previous studies that further characterize racial and gender differences in the experiences with racial and weight discrimination in a robust sample of ethnically diverse adults with obesity. Asking participants in pain and weight management interventions about their experiences with racial or weight discrimination could help clinicians make culturally informed decisions that address barriers to pain relief and weight loss. Future studies should build on these findings by investigating whether training providers to ask about and validate experiences of racial and weight discrimination has prognostic and therapeutic benefits. Additionally, clinicians and researchers could collaboratively develop and clinically validate intervention targets that account for frequent experiences with racial and weight discrimination.

\section{Declarations}

Ethics approval: All study procedures were approved by the Institutional Review Boards at NYU School of Medicine (\#i16-01445), VA New York Harbor (\#01624), and Albert Einstein College of Medicine in collaboration with the Montefiore Health System (\#2017-7603). All methods were performed in accordance with the relevant guidelines and regulations. Written informed consent was obtained by all participants prior to any data collection.

Consent for publication: Not applicable.

Availability of data and materials: The datasets and materials used and/or analysed during the current study available from the corresponding author on reasonable request.

Competing Interests: The authors declare no competing interests.

Funding: This research was funded by the National Institutes of Health $(\mathrm{NIH})$, National Institute of Diabetes and Digestive and Kidney Diseases [1R01DK111928]; National Center for Advancing Translational Sciences [1UL1 TR001445]; a Career Development Award [HX000762- 01A1] through the Veterans Affairs Health Services Research and Development Service; and NY Regional Center for Diabetes Translational Research (CDTR) [P30DK111022]. The funding sources had no role in study design, data collection, data analysis, interpretation of data, writing of the report, and in the decision to submit the article for publication.

Author Contributions: EM, MJ, and SW developed the study hypotheses and study design. SW and EB performed the data collection. BW and SW performed the data analysis and interpretation under the guidance of of EM, MJ, and JR. EM, SW, GC, EB, and RT drafted the manuscript, and MJ, NB, and JR provided critical revisions. All authors approved the final version of the paper for submission. 
Acknowledgements: We express deep gratitude to our research staff for their many contributions to the project: Adefunke Ajenikoko, Lorena Velastegui, Rosalie, Grullon, Alia Dixon, Kevin Salinas, and Brianna Bhoopsingh. We also thank Claudia Lechuga, Mariel Connolly, Dr. Jeannette Beasley, Dr. Nicole Hollingsworth, Melinda Marquez, and Clare Viglione. We also thank the research interns and the clinical staff at the VA New York Harbor Healthcare System (Manhattan campus) and Montefiore Medical Group practices - Bronx East, Castle Hill, Grand Concourse, and University Avenue - for their support. Not least of all, we would like to especially thank all of the patients and providers participating in the GEM study.

\section{References}

1. Edwards RR. The association of perceived discrimination with low back pain. J Behav Med [Internet]. 2008 Oct [cited 2021 Apr 9];31(5):379-89. Available from:

https://pubmed.ncbi.nlm.nih.gov/18581224/

2. Brown TT, Partanen J, Chuong L, Villaverde V, Chantal Griffin A, Mendelson A. Discrimination hurts: The effect of discrimination on the development of chronic pain. Soc Sci Med. 2018;204(March):18.

3. Bakhshaie J, Rogers AH, Mayorga NA, Ditre J, Rodríguez-Cano R, Ruiz AC, et al. Perceived Racial Discrimination and Pain Intensity/Disability Among Economically Disadvantaged Latinos in a Federally Qualified Health Center: The Role of Anxiety Sensitivity. J Immigr Minor Heal [Internet]. 2019 Feb 15 [cited 2021 Apr 9];21(1):21-9. Available from:

https://pubmed.ncbi.nlm.nih.gov/29460134/

4. Hales CM, Carroll MD, Fryar CD, Ogden CL. Prevalence of Obesity Among Adults and Youth: United States, 2015-2016. NCHS Data Brief. 2017;(288):1-8.

5. Narouze S, Souzdalnitski D. Obesity and chronic pain: Systematic review of prevalence and implications for pain practice [Internet]. Vol. 40, Regional Anesthesia and Pain Medicine. Lippincott Williams and Wilkins; 2015 [cited 2021 Apr 10]. p. 91-111. Available from:

https://pubmed.ncbi.nlm.nih.gov/25650632/

6. Stone AA, Broderick JE. Obesity and pain are associated in the United States. Obesity [Internet]. 2012 [cited 2021 Apr 10];20(7):1491-5. Available from: https://pubmed.ncbi.nlm.nih.gov/22262163/

7. Zhang TT, Liu Z, Liu YL, Zhao JJ, Liu DW, Tian QB. Obesity as a Risk Factor for Low Back Pain [Internet]. Vol. 31, Clinical Spine Surgery. Lippincott Williams and Wilkins; 2018 [cited 2021 Apr 10]. p. 22-7. Available from: https://pubmed.ncbi.nlm.nih.gov/27875413/

8. Cooper L, Ells L, Ryan C, Martin D. Perceptions of adults with overweight/obesity and chronic musculoskeletal pain: An interpretative phenomenological analysis. J Clin Nurs [Internet]. 2018 Mar 1 [cited 2021 Apr 10];27(5-6):e776-86. Available from: https://pubmed.ncbi.nlm.nih.gov/29148620/

9. Burgess DJ, Grill J, Noorbaloochi S, Griffin JM, Ricards J, Van Ryn M, et al. The effect of perceived racial discrimination on bodily pain among older African American men. Pain Med [Internet]. 2009 Nov [cited 2021 Apr 9];10(8):1341-52. Available from: https://pubmed.ncbi.nlm.nih.gov/20021596/ 
10. Goodin BR, Glover TL, King CD, Sibille KT, Cruz-Almeida Y, Staud R, et al. Perceived racial discrimination, but not mistrust of medical researchers, predicts the heat pain tolerance of african americans with symptomatic knee osteoarthritis. Heal Psychol [Internet]. 2013 Nov [cited 2021 Apr 9];32(11):1117-26. Available from: /pmc/articles/PMC3943939/

11. Puhl R, Brownell KD. Bias, discrimination, and obesity [Internet]. Vol. 9, Obesity Research. North American Assoc. for the Study of Obesity; 2001 [cited 2021 Apr 9]. p. 788-805. Available from: https://pubmed.ncbi.nlm.nih.gov/11743063/

12. Puhl RM, Heuer CA. The Stigma of Obesity: A Review and Update. Obesity [Internet]. 2009 May 1 [cited 2021 Apr 9];17(5):941-64. Available from: http://doi.wiley.com/10.1038/oby.2008.636

13. Puhl RM, Andreyeva T, Brownell KD. Perceptions of weight discrimination: Prevalence and comparison to race and gender discrimination in America. Int J Obes [Internet]. 2008 Jun [cited 2021 Apr 9];32(6):992-1000. Available from: https://pubmed.ncbi.nlm.nih.gov/18317471/

14. Schafer MH, Ferraro KF. The stigma of obesity: Does perceived weight discrimination affect identity and physical health? Soc Psychol Q [Internet]. 2011 Mar [cited 2021 Apr 9];74(1):76-97. Available from: http://spq.sagepub.com

15. Hatzenbuehler ML, Keyes KM, Hasin DS. Associations between perceived weight discrimination and the prevalence of psychiatric disorders in the general population. Obesity [Internet]. 2009 Nov [cited 2021 Apr 9];17(11):2033-9. Available from: /pmc/articles/PMC3767420/

16. Jackson SE, Beeken RJ, Wardle J. Perceived weight discrimination and changes in weight, waist circumference, and weight status. Obesity [Internet]. 2014 Dec 1 [cited 2021 Apr 9];22(12):2485-8. Available from: https://pubmed.ncbi.nlm.nih.gov/25212272/

17. Sutin AR, Terracciano A. Perceived Weight Discrimination and Obesity. Newton RL, editor. PLoS One [Internet]. 2013 Jul 24 [cited 2021 Apr 9];8(7):e70048. Available from: https://dx.plos.org/10.1371/journal.pone.0070048

18. Latner JD, Barile JP, Durso LE, O’Brien KS. Weight and health-related quality of life: The moderating role of weight discrimination and internalized weight bias. Eat Behav [Internet]. 2014 Dec 1 [cited 2021 Apr 9];15(4):586-90. Available from: https://pubmed.ncbi.nlm.nih.gov/25215477/

19. Sutin AR, Stephan Y, Terracciano A. Weight Discrimination and Risk of Mortality. Psychol Sci [Internet]. 2015 Nov 1 [cited 2020 Jul 28];26(11):1803-11. Available from: https://pubmed.ncbi.nlm.nih.gov/26420442/

20. Schmitt MT, Postmes T, Branscombe NR, Garcia A. The consequences of perceived discrimination for psychological well-being: A meta-analytic review. Psychol Bull [Internet]. 2014 [cited 2021 Apr 9];140(4):921-48. Available from: https://psycnet.apa.org/journals/bul/140/4/921

21. Udo T, Grilo CM. Cardiovascular disease and perceived weight, racial, and gender discrimination in U.S. adults. J Psychosom Res. 2017 Sep 1;100:83-8.

22. Brodish AB, Cogburn CD, Fuller-Rowell TE, Peck S, Malanchuk O, Eccles JS. Perceived Racial Discrimination as a Predictor of Health Behaviors: The Moderating Role of Gender. Race Soc Probl 
[Internet]. 2011 Oct 1 [cited 2021 Apr 9];3(3):160-9. Available from:

https://pubmed.ncbi.nlm.nih.gov/22844386/

23. Terry EL, Fullwood MD, Booker SQ, Cardoso JS, Sibille KT, Glover TL, et al. Everyday discrimination in adults with knee pain: The role of perceived stress and pain catastrophizing. J Pain Res [Internet]. 2020 [cited 2021 Apr 9];13:883-95. Available from: /pmc/articles/PMC7200232/

24. Mwendwa DT, Gholson G, Sims RC, Levy SA, Ali M, Harrell CJ, et al. Coping with perceived racism: A significant factor in the development of obesity in African American women? J Natl Med Assoc [Internet]. 2011 [cited 2021 Apr 9];103(7):602-8. Available from: /pmc/articles/PMC5003024/

25. Dutton GR, Lewis TT, Durant N, Halanych J, Kiefe Cl, Sidney S, et al. Perceived weight discrimination in the CARDIA study: Differences by race, sex, and weight status. Obesity [Internet]. 2014 Feb [cited 2021 Apr 9];22(2):530-6. Available from: /pmc/articles/PMC3695009/

26. Moore, Cooper, Davis-Smith. African American Obese Mens' Attitudes and Perceptions of Bariatric Surgery: A Phenomenological Study. Spectr A J Black Men. 2016;4(2):43.

27. Smith ED, Layden BT, Hassan C, Sanchez-Johnsen L. Surgical Treatment of Obesity in Latinos and African Americans: Future Directions and Recommendations to Reduce Disparities in Bariatric Surgery [Internet]. Vol. 13, Bariatric Surgical Practice and Patient Care. Mary Ann Liebert Inc.; 2018 [cited 2021 Jun 2]. p. 2-11. Available from: https://www.liebertpub.com/doi/abs/10.1089/bari.2017.0037

28. Torensma B, Thomassen I, van Velzen M, in 't Veld BA. Pain Experience and Perception in the Obese Subject Systematic Review (Revised Version) [Internet]. Vol. 26, Obesity Surgery. Springer New York LLC; 2016 [cited 2021 Apr 9]. p. 631-9. Available from: https://pubmed.ncbi.nlm.nih.gov/26661107/

29. Guh DP, Zhang W, Bansback N, Amarsi Z, Birmingham CL, Anis AH. The incidence of co-morbidities related to obesity and overweight: A systematic review and meta-analysis. BMC Public Health [Internet]. 2009 Dec 25 [cited 2021 Apr 9];9(1):88. Available from: http://bmcpublichealth.biomedcentral.com/articles/10.1186/1471-2458-9-88

30. Wittleder S, Ajenikoko A, Bouwman D, Fang Y, McKee MD, Meissner P, et al. Protocol for a clusterrandomized controlled trial of a technology-assisted health coaching intervention for weight management in primary care: The GEM (goals for eating and moving) study. Contemp Clin Trials [Internet]. 2019;83(January):37-45. Available from: https://doi.org/10.1016/j.cct.2019.06.005

31. Maayan L, Correll CU. Management of Antipsychotic-Related Weight Gain \$watermark-text \$watermark-text \$watermark-text. Expert Rev Neurother. 2010;10(7):1175-200.

32. Krieger N, Smith K, Naishadham D, Hartman C, Barbeau EM. Experiences of discrimination: Validity and reliability of a self-report measure for population health research on racism and health. Soc Sci Med [Internet]. 2005 Oct [cited 2019 Sep 19];61(7):1576-96. Available from: http://www.ncbi.nlm.nih.gov/pubmed/16005789

33. Herbert MS, Goodin BR, Bulls HW, Sotolongo A, Petrov ME, Edberg JC, et al. Ethnicity, cortisol, and experimental pain responses among persons with symptomatic knee osteoarthritis. Clin J Pain [Internet]. 2017 [cited 2021 Apr 9];33(9):820-6. Available from: /pmc/articles/PMC5446937/ 
34. Cuevas AG, Ortiz K, Ransome Y. The moderating role of race/ethnicity and nativity in the relationship between perceived discrimination and overweight and obesity: Results from the National Epidemiologic Survey on Alcohol and Related Conditions. BMC Public Health [Internet]. 2019 Nov 6 [cited 2021 Apr 9];19(1). Available from: /pmc/articles/PMC6833296/

35. Udo T, Grilo CM. Cardiovascular disease and perceived weight, racial, and gender discrimination in U.S. adults. J Psychosom Res [Internet]. 2017 Sep 1 [cited 2021 Apr 9];100:83-8. Available from: /pmc/articles/PMC5598460/

36. Udo T, Purcell K, Grilo CM. Perceived weight discrimination and chronic medical conditions in adults with overweight and obesity. Int J Clin Pract [Internet]. 2016 Dec 1 [cited 2019 Sep 19];70(12):100311. Available from: http://www.ncbi.nlm.nih.gov/pubmed/28032427

37. Salem R, Bamer AM, Alschuler KN, Johnson KL, Amtmann D. Obesity and symptoms and quality of life indicators of individuals with disabilities. In: Disability and Health Journal. Elsevier; 2014. p. 124-30.

38. Stone AL, Holley AL, Dieckmann NF, Wilson AC. Use of the PROMIS-29® to identify subgroups of mothers with chronic pain. Heal Psychol. 2019 May 1;38(5):422-30.

39. Lans J, Baker DJ, Castelein RM, Sood RF, Chen NC, Eberlin KR. Patient-reported outcomes following surgical treatment of symptomatic digital neuromas. Plast Reconstr Surg [Internet]. 2020 [cited 2021 Apr 9];145(3):563E-573E. Available from: https://pubmed.ncbi.nlm.nih.gov/32097316/

40. Hageman PA, Mroz JE, Yoerger MA, Pullen $\mathrm{CH}$. Weight loss is associated with improved quality of life among rural women completers of a web-based lifestyle intervention. PLoS One [Internet]. 2019 Nov 1 [cited 2021 Apr 9];14(11). Available from: https://pubmed.ncbi.nlm.nih.gov/31743365/

41. Cornelio-Flores O, Lestoquoy AS, Abdallah S, Deloureiro A, Lorente K, Pardo B, et al. The latino integrative medical group visit as a model for pain reduction in underserved Spanish speakers. $J$ Altern Complement Med [Internet]. 2018 Feb 1 [cited 2021 Apr 9];24(2):125-31. Available from: https://pubmed.ncbi.nlm.nih.gov/28885858/

42. LaVela SL, Etingen B, Miskevics S, Cella D. Use of PROMIS-29® in US Veterans: Diagnostic Concordance and Domain Comparisons with the General Population. J Gen Intern Med [Internet]. 2019 Aug 15 [cited 2021 Apr 9];34(8):1452-8. Available from: https://pubmed.ncbi.nlm.nih.gov/31144276/

43. Gardiner P, D’Amico S, Luo M, Haas N. An innovative electronic health toolkit (Our Whole Lives for Chronic Pain) to reduce chronic pain in patients with health disparities: Open clinical trial. JMIR mHealth uHealth [Internet]. 2020 Mar 1 [cited 2021 Apr 9];8(3). Available from: https://pubmed.ncbi.nlm.nih.gov/32224487/

44. Cella D, Choi SW, Condon DM, Schalet B, Hays RD, Rothrock NE, et al. PROMIS® Adult Health Profiles: Efficient Short-Form Measures of Seven Health Domains. Value Heal. 2019 May 1;22(5):537-44.

45. Jackson SE, Steptoe A. Association between perceived weight discrimination and physical activity: A population-based study among English middle-aged and older adults. BMJ Open [Internet]. 2017 Mar 1 [cited 2021 Jun 3];7(3):14592. Available from: http://bmjopen.bmj.com/ 
46. Brody GH, Yu T, Chen E, Ehrlich KB, Miller GE. Racial Discrimination, Body Mass Index, and Insulin Resistance: A Longitudinal Analysis. Heal Psychol [Internet]. 2018 Dec 1 [cited 2021 Jun 3];37(12):1107-14. Available from: / pmc/articles/PMC6277234/

47. Croft P, Blyth FM, Van Der Windt D. Chronic Pain Epidemiology: From Aetiology to Public Health. Chronic Pain Epidemiology: From Aetiology to Public Health. Oxford University Press; 2011. 1-384 p.

48. Dailey DL, Vance CGT, Rakel BA, Zimmerman MB, Embree J, Merriwether EN, et al. Transcutaneous Electrical Nerve Stimulation Reduces Movement-Evoked Pain and Fatigue: A Randomized, Controlled Trial. Arthritis Rheumatol [Internet]. 2020 May 1 [cited 2021 Jun 3];72(5):824-36. Available from: https://pubmed.ncbi.nlm.nih.gov/31738014/

49. Dahlhamer J, Lucas J, Zelaya, C, Nahin R, Mackey S, DeBar L, et al. Prevalence of Chronic Pain and High-Impact Chronic Pain Among Adults - United States, 2016. MMWR Morb Mortal Wkly Rep [Internet]. 2018 Sep 14 [cited 2021 Apr 9];67(36):1001-6. Available from: http://www.cdc.gov/mmwr/volumes/67/wr/mm6736a2.htm?s_cid=mm6736a2_w

50. Godfrey KM, Bullock AJ, Dorflinger LM, Min KM, Ruser CB, Masheb RM. Pain and modifiable risk factors among weight loss seeking Veterans with overweight. Appetite [Internet]. 2018 Sep 1 [cited 2021 Apr 9];128:100-5. Available from: https://pubmed.ncbi.nlm.nih.gov/29885382/

51. Byrd AS, Toth AT, Stanford FC. Racial Disparities in Obesity Treatment [Internet]. Vol. 7, Current obesity reports. NIH Public Access; 2018 [cited 2021 Jun 2]. p. 130-8. Available from: /pmc/articles/PMC6066592/

52. Roshania R, Narayan KMV, Oza-Frank R. Age at arrival and risk of obesity among US immigrants. Obesity [Internet]. 2008 Dec [cited 2021 Apr 9];16(12):2669-75. Available from: https://pubmed.ncbi.nlm.nih.gov/18846044/

53. Edwards RR, Doleys DM, Fillingim RB, Lowery D. Ethnic differences in pain tolerance: Clinical implications in a chronic pain population. Psychosom Med [Internet]. 2001 [cited $2021 \mathrm{Apr}$ 9];63(2):316-23. Available from: https://pubmed.ncbi.nlm.nih.gov/11292281/

54. Rahim-Williams FB, Riley JL, Herrera D, Campbell CM, Hastie BA, Fillingim RB. Ethnic identity predicts experimental pain sensitivity in African Americans and Hispanics. Pain [Internet]. 2007 May [cited 2021 Apr 9];129(1-2):177-84. Available from: https://pubmed.ncbi.nlm.nih.gov/17296267/

55. Cruz-Almeida Y, Sibille KT, Goodin BR, Petrov ME, Bartley EJ, Riley JL, et al. Racial and Ethnic Differences in Older Adults With Knee Osteoarthritis. Arthritis Rheumatol [Internet]. 2014 Jul 1 [cited 2021 Apr 9];66(7):1800-10. Available from: http://doi.wiley.com/10.1002/art.38620

56. Riley JL, Wade JB, Myers CD, Sheffield D, Papas RK, Price DD. Racial/ethnic differences in the experience of chronic pain. Pain [Internet]. 2002 Dec [cited 2021 Apr 9];100(3):291-8. Available from: https://pubmed.ncbi.nlm.nih.gov/12468000/

57. Zettel-Watson L, Rutledge DN, Aquino JK, Cantero P, Espinoza A, Leal F, et al. Typology of chronic pain among overweight Mexican Americans. J Health Care Poor Underserved [Internet]. 2011 [cited 2021 Apr 9];22(3):1030-47. Available from: https://pubmed.ncbi.nlm.nih.gov/21841294/ 
58. Reyes-Gibby CC, Aday LA, Todd KH, Cleeland CS, Anderson KO. Pain in Aging Community-Dwelling Adults in the United States: Non-Hispanic Whites, Non-Hispanic Blacks, and Hispanics. J Pain [Internet]. 2007 Jan [cited 2021 Apr 9];8(1):75-84. Available from:

https://pubmed.ncbi.nlm.nih.gov/16949874/

59. Dugan SA, Lewis TT, Everson-Rose SA, Jacobs EA, Harlow SD, Janssen I. Chronic discrimination and bodily pain in a multiethnic cohort of midlife women in the Study of Women's Health Across the Nation. Pain. 2017;158(9):1656-65.

60. Dunlevy C, MacLellan GA, O’Malley E, Blake C, Breen C, Gaynor K, et al. Does changing weight change pain? Retrospective data analysis from a national multidisciplinary weight management service. Eur J Pain [Internet]. 2019 Sep 21 [cited 2021 Apr 9];23(8):1403-15. Available from: https://onlinelibrary.wiley.com/doi/abs/10.1002/ejp.1397

61. Williams DR, Neighbors HW, Jackson JS. Racial/Ethnic Discrimination and Health: Findings From Community Studies. Am J Public Health [Internet]. 2003 Feb 1 [cited 2021 Apr 9];93(2):200-8. Available from: http://ajph.aphapublications.org/doi/10.2105/AJPH.93.2.200

62. Pascoe EA, Richman LS. Perceived Discrimination and Health: A Meta-Analytic Review. Psychol Bull [Internet]. 2009 Jul [cited 2021 Apr 9];135(4):531-54. Available from: /pmc/articles/PMC2747726/

63. Gee GC, Spencer MS, Chen J, Takeuchi D. A nationwide study of discrimination and chronic health conditions among Asian Americans. Am J Public Health [Internet]. 2007 Jan 7 [cited 2021 Apr 9];97(7):1275-82. Available from: /pmc/articles/PMC1913081/

64. Campbell CM, France CR, Robinson ME, Logan HL, Geffken GR, Fillingim RB. Ethnic Differences in Diffuse Noxious Inhibitory Controls. J Pain [Internet]. 2008 Aug [cited 2021 Apr 9];9(8):759-66. Available from: https://pubmed.ncbi.nlm.nih.gov/18482870/

65. Rahim-Williams B, Riley JL, Williams AKK, Fillingim RB. A Quantitative Review of Ethnic Group Differences in Experimental Pain Response: Do Biology, Psychology, and Culture Matter? [Internet]. Vol. 13, Pain Medicine. Blackwell Publishing Inc.; 2012 [cited 2021 Apr 9]. p. 522-40. Available from: https://pubmed.ncbi.nlm.nih.gov/22390201/

66. Crombez G, Van Damme S, Eccleston C. Hypervigilance to pain: An experimental and clinical analysis [Internet]. Vol. 116, Pain. Pain; 2005 [cited 2021 Apr 9]. p. 4-7. Available from: https://pubmed.ncbi.nlm.nih.gov/15927387/

67. Hollingshead NA, Ashburn-Nardo L, Stewart JC, Hirsh AT. The Pain Experience of Hispanic Americans: A Critical Literature Review and Conceptual Model [Internet]. Vol. 17, Journal of Pain. Churchill Livingstone Inc.; 2016 [cited 2021 Apr 9]. p. 513-28. Available from: https://pubmed.ncbi.nlm.nih.gov/26831836/

68. Nguyen M, Ugarte C, Fuller I, Haas G, Portenoy RK. Access to care for chronic pain: Racial and ethnic differences. J Pain. 2005 May 1;6(5):301-14.

69. Hacker K, Chu J, Leung C, Marra R, Pirie A, Brahimi M, et al. The impact of Immigration and Customs Enforcement on immigrant health: Perceptions of immigrants in Everett, Massachusetts, USA. Soc 
Sci Med [Internet]. 2011 Aug [cited 2021 Apr 9];73(4):586-94. Available from: https://pubmed.ncbi.nlm.nih.gov/21778008/

70. Villarruel AM. Mexican-American cultural meanings, expressions, self-care and dependent-care actions associated with experiences of pain. Res Nurs Health [Internet]. 1995 Oct 1 [cited 2021 Apr 9];18(5):427-36. Available from: http://doi.wiley.com/10.1002/nur.4770180508

71. Wiesenfeld-Hallin Z. Sex differences in pain perception [Internet]. Vol. 2, Gender Medicine. Gend Med; 2005 [cited 2021 Apr 9]. p. 137-45. Available from: https://pubmed.ncbi.nlm.nih.gov/16290886/

72. Unruh AM. Gender variations in clinical pain experience [Internet]. Vol. 65, Pain. Elsevier B.V.; 1996 [cited 2021 Apr 9]. p. 123-67. Available from: https://pubmed.ncbi.nlm.nih.gov/8826503/

73. Soetanto ALF, Chung JWY, Wong TKS. Are there gender differences in pain perception? J Neurosci Nurs [Internet]. 2006 Jun [cited 2021 Apr 9];38(3):172-6. Available from: https://pubmed.ncbi.nlm.nih.gov/16817669/

74. Andersson HI, Ejlertsson G, Leden I, Rosenberg C. Chronic pain in a geographically defined general population: Studies of differences in age, gender, social class, and pain localization. Clin J Pain [Internet]. 1993 Sep 1 [cited 2021 Jun 2];9(3):174-82. Available from: https://europepmc.org/article/med/8219517

75. Kroenke K, Spitzer RL. Gender differences in the reporting of physical and somatoform symptoms. Psychosom Med [Internet]. 1998 [cited 2021 Apr 9];60(2):150-5. Available from: https://pubmed.ncbi.nlm.nih.gov/9560862/

76. Kroenke K, Price RK. Symptoms in the Community: Prevalence, Classification, and Psychiatric Comorbidity. Arch Intern Med [Internet]. 1993 Nov 8 [cited 2021 Apr 9];153(21):2474-80. Available from: https://jamanetwork.com/

77. Jackson JL, Chamberlin J, Kroenke K. Gender and symptoms in primary care practices [Internet]. Vol. 44, Psychosomatics. American Psychiatric Publishing Inc.; 2003 [cited 2021 Apr 9]. p. 359-66. Available from: https://pubmed.ncbi.nlm.nih.gov/12954909/

78. Barsky AJ, Peekna HM, Borus JF. Somatic symptom reporting in women and men. J Gen Intern Med [Internet]. 2001 [cited 2021 Apr 9];16(4):266-26675. Available from:

https://pubmed.ncbi.nlm.nih.gov/11318929/

79. Scudds RJ, McD. Robertson J. Empirical evidence of the association between the presence of musculoskeletal pain and physical disability in community-dwelling senior citizens. Pain [Internet]. 1998 Jan [cited 2021 Apr 9];75(2-3):229-35. Available from:

https://pubmed.ncbi.nlm.nih.gov/9583758/

80. Ochroch EA, Gottschalk A, Troxel AB, Farrar JT. Women suffer more short and long-term pain than men after major thoracotomy. Clin J Pain [Internet]. 2006 Jun [cited 2021 Apr 9];22(5):491-8. Available from: https://pubmed.ncbi.nlm.nih.gov/16772805/

81. Ng KFJ, Tsui SL, Chan WS. Prevalence of common chronic pain in Hong Kong adults. Clin J Pain [Internet]. 2002 [cited 2021 Apr 9];18(5):275-81. Available from: https://pubmed.ncbi.nlm.nih.gov/12218497/ 
82. Fillingim RB. Sex, gender, and pain: women and men really are different. [Internet]. Vol. 4, Current review of pain. Curr Rev Pain; 2000 [cited 2021 Apr 9]. p. 24-30. Available from:

https://pubmed.ncbi.nlm.nih.gov/10998712/

83. Stubbs DW, Krebs E, Bair M, Damush T, Wu J, Sutherland J, et al. Sex differences in pain and painrelated disability among primary care patients with chronic musculoskeletal pain. Pain Med [Internet]. 2010 [cited 2021 Apr 9];11(2):232-9. Available from:

https://pubmed.ncbi.nlm.nih.gov/20002591/

84. Poleshuck EL, Giles DE, Tu X. Pain and depressive symptoms among financially disadvantaged women's health patients. J Women's Heal [Internet]. 2006 Mar [cited 2021 Apr 9];15(2):182-93. Available from: https://pubmed.ncbi.nlm.nih.gov/16536682/

85. Robinson ME, Dannecker EA, George SZ, Otis J, Atchison JW, Fillingim RB. Sex differences in the associations among psychological factors and pain report: A novel psychophysical study of patients with chronic low back pain. J Pain [Internet]. 2005 Jul [cited 2021 Apr 9];6(7):463-70. Available from: https://pubmed.ncbi.nlm.nih.gov/15993825/

86. Keogh E, McCracken LM, Eccleston C. Do men and women differ in their response to interdisciplinary chronic pain management? Pain [Internet]. 2005 [cited 2021 Apr 9];114(1-2):37-46. Available from: https://pubmed.ncbi.nlm.nih.gov/15733629/

87. Rhudy JL, Williams AE. Gender differences in pain: Do emotions play a role? [Internet]. Vol. 2, Gender Medicine. Gend Med; 2005 [cited 2021 Apr 9]. p. 208-26. Available from:

https://pubmed.ncbi.nlm.nih.gov/16464733/

88. Chesterton LS, Barlas P, Foster NE, Baxter GD, Wright CC. Gender differences in pressure pain threshold in healthy humans. Pain [Internet]. 2003 [cited 2021 Apr 9];101(3):259-66. Available from: https://pubmed.ncbi.nlm.nih.gov/12583868/

89. Keogh E, Herdenfeldt M. Gender, coping and the perception of pain. Pain [Internet]. 2002 [cited 2021 Apr 9];97(3):195-201. Available from: https://pubmed.ncbi.nlm.nih.gov/12044616/

90. Greenspan JD, Craft RM, LeResche L, Arendt-Nielsen L, Berkley KJ, Fillingim RB, et al. Studying sex and gender differences in pain and analgesia: A consensus report [Internet]. Vol. 132, Pain. Pain; 2007 [cited 2021 Apr 9]. Available from: https://pubmed.ncbi.nlm.nih.gov/17964077/

91. Wijnhoven HAH, De Vet HCW, Picavet HSJ. Sex differences in consequences of musculoskeletal pain. Spine (Phila Pa 1976) [Internet]. 2007 May [cited 2021 Apr 9];32(12):1360-7. Available from: https://pubmed.ncbi.nlm.nih.gov/17515827/

92. Wijnhoven HAH, de Vet HCW, Picavet HSJ. Explaining sex differences in chronic musculoskeletal pain in a general population. Pain [Internet]. 2006 Sep [cited 2021 Apr 9];124(1-2):158-66. Available from: https://pubmed.ncbi.nlm.nih.gov/16716517/

93. Quartana PJ, Campbell CM, Edwards RR. Pain catastrophizing a critical review [Internet]. Vol. 9, Expert Review of Neurotherapeutics. NIH Public Access; 2009 [cited 2021 Apr 9]. p. 745-58. Available from: /pmc/articles/PMC2696024/ 
94. Pearl RL, Wadden TA, Tronieri JS, Chao AM, Alamuddin N, Berkowitz RI. Everyday discrimination in a racially diverse sample of patients with obesity. Clin Obes [Internet]. 2018 Apr 1 [cited 2021 Apr 9];8(2):140-6. Available from: https://pubmed.ncbi.nlm.nih.gov/29266824/

95. Gee GC, Ro A, Gavin A, Takeuchi DT. Disentangling the effects of racial and weight discrimination on body mass index and obesity among Asian Americans. Am J Public Health [Internet]. 2008 Mar 1 [cited 2021 Apr 9];98(3):493-500. Available from: /pmc/articles/PMC2253588/

96. Mehok LE, Miller MM, Trost Z, Goubert L, De Ruddere L, Hirsh AT. Pain intensity and attribution mediate the impact of patient weight and gender on activity recommendations for chronic pain. $J$ Pain Res [Internet]. 2019 [cited 2021 Apr 9];12:2743-53. Available from: https://pubmed.ncbi.nlm.nih.gov/31571978/

\section{Figures}


a)

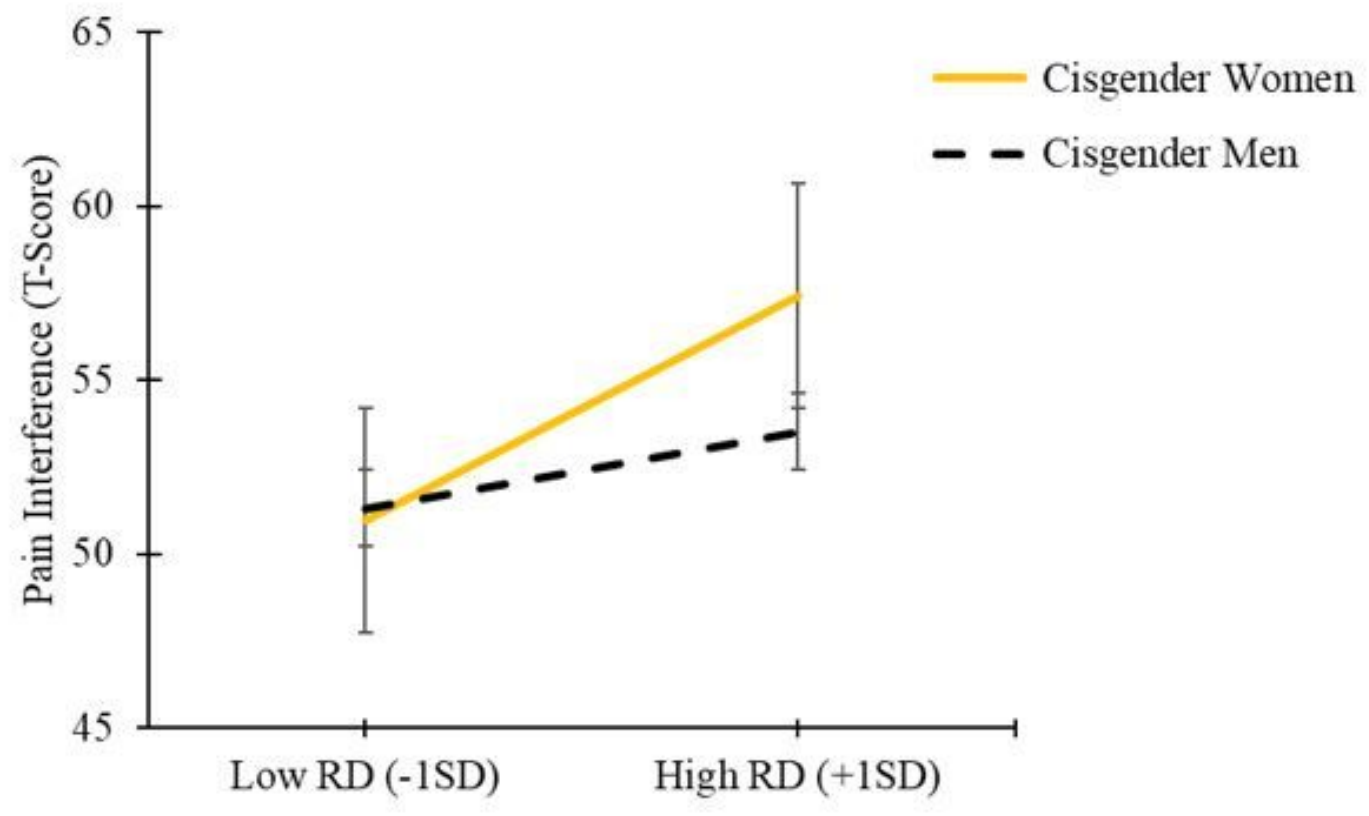

b)

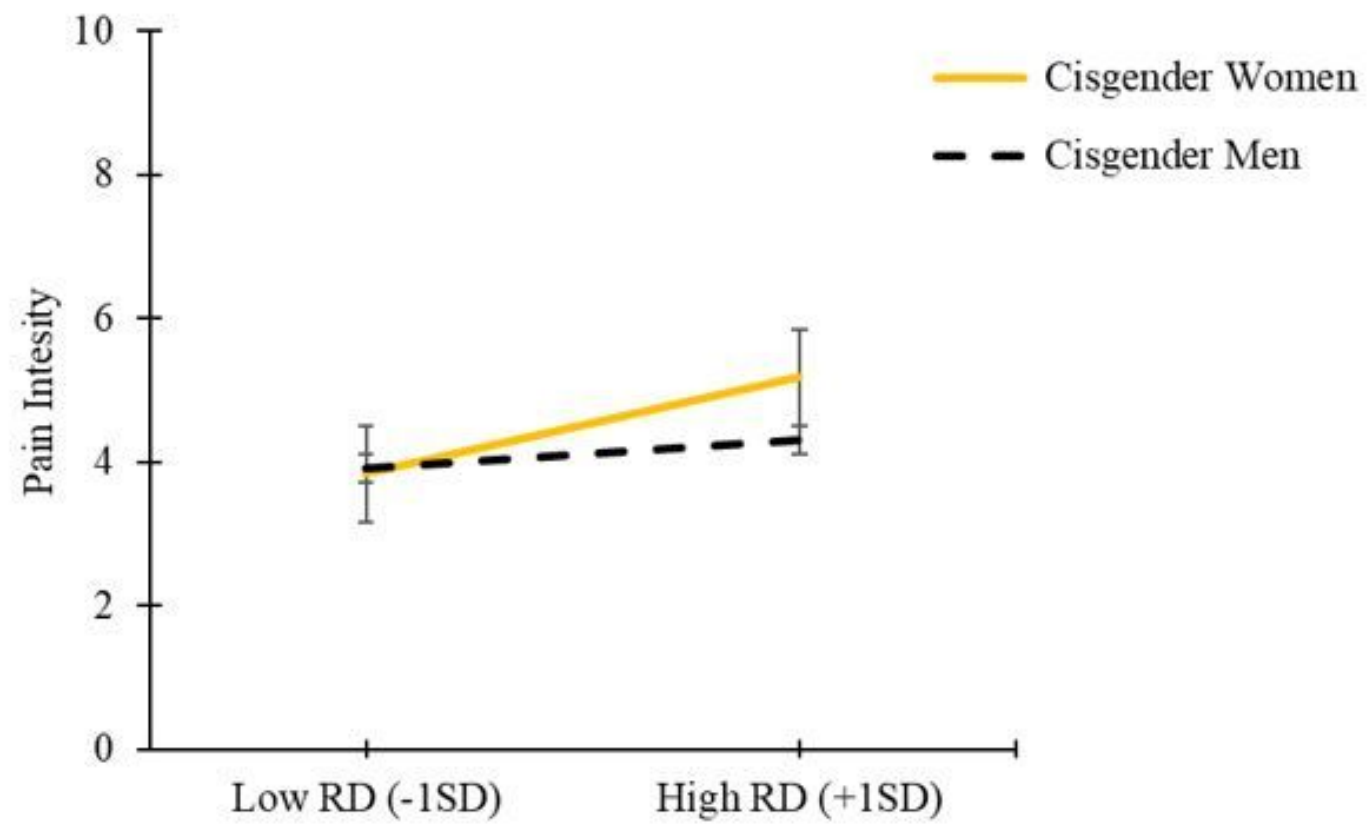

\section{Figure 1}

Pain interference scores (a) and pain intensity scores (b) by experiences of racial discrimination for selfidentified gender groups Note. Pain interference T-scores (a) and pain intensity scores (b) of participants who self-identified as male (cisgender men) or female (cisgender women) are shown for low (-1 SD) and high (+ $1 \mathrm{SD}$ ) self-reported racial discrimination (RD). 


\section{Supplementary Files}

This is a list of supplementary files associated with this preprint. Click to download.

- MerriwetherPainDiscriminationSupp06022021.docx 